TUM-HEP-291/97

July 1997

\title{
SUSY in Rare and CP-Violating $B$ Decays
}

\author{
A. MASIERO \\ SISSA - ISAS, Trieste and Dip. di Fisica, Università di Perugia and INFN, \\ Sezione di Perugia, Via Pascoli, I-06100 Perugia, Italy \\ L. SILVESTRINI \\ Physik Department, Technische Universität München, D-85748 Garching, Germany \\ In this talk we discuss rare $B$ decays $\left(b \rightarrow s \gamma, b \rightarrow s g, b \rightarrow s \ell^{+} \ell^{-}\right), B-\bar{B}$ \\ oscillations and CP violation in $B$ physics in the context of low-energy SUSY. We \\ outline the variety of predictions that arise according to the choice of the SUSY \\ extension ranging from what we call the "minimal" version of the MSSM to models \\ without flavour universality or with broken R-parity. In particular, we provide a \\ model-independent parameterization of the SUSY FCNC and CP-violating effects \\ which is useful in tackling the problem in generic low-energy SUSY. We show how \\ rare $B$ decays and $\mathrm{CP}$ violation in $B$-decay amplitudes may be complementary to \\ direct SUSY searches at colliders, in particular for what concerns extensions of the \\ most restrictive version of the MSSM.
}

\section{Introduction}

The generation of fermion masses and mixings ("flavour problem") gives rise to a first and important distinction among theories of new physics beyond the electroweak Standard Model (SM). Indeed, one may conceive a kind of new physics which is completely "flavour blind", i.e. new interactions which have nothing to do with the flavour structure. To provide an example of such a situation, consider a scheme where flavour arises at a very large scale (for instance the Planck mass) while new physics is represented by a supersymmetric (SUSY) extension of the SM with supersymmetry broken at a much lower scale and with the SUSY breaking transmitted to the observable sector by flavour blind gauge interactions. In this case one may think that the new physics does not cause any major change to the original flavour structure of the SM, namely that the pattern of fermion masses and mixings is compatible with the numerous and demanding tests of Flavour Changing Neutral Currents (FCNC).

${ }^{a}$ Talk given by A. Masiero at the 2nd International Conference on B Physics and CP Violation (BCONF 97), Honolulu, HI, 24-28 Mar 1997. 
Alternatively, one can conceive a new physics which is entangled with the flavour problem. As an example consider a technicolour scheme where fermion masses and mixings arise through the exchange of new gauge bosons which mix together ordinary and technifermions. Here we expect (correctly enough) new physics to have potential problems in accommodating the usual fermion spectrum with the adequate suppression of FCNC. As another example of new physics which is not flavour blind, take a more conventional SUSY model which is derived from a spontaneously broken $\mathrm{N}=1$ supergravity and where the SUSY breaking information is conveyed to the ordinary sector of the theory through gravitational interactions. In this case we may expect that the scale at which flavour arises and the scale of SUSY breaking are not so different and possibly the mechanism itself of SUSY breaking and transmission is flavour-dependent. Under these circumstances we may again expect a potential flavour problem to arise, namely that SUSY contributions to FCNC processes are too large.

The potentiality of probing SUSY in FCNC phenomena was reafily realized when the era of SUSY phenomenology started in the early 80's In particular, the major implication that the scalar partners of quarks of the same electric charge but belonging to different generations had to share a remarkably high mass degeneracy was emphasized.

Throughout the large amount of work in this last decade it became clearer and clearer that generically talking of the implications of low-energy SUSY on FCNC may be rather misleading. We have a minimal SUSY extension of the SM, the so-called Minimal Supersymmetric Standard Model (MSSM) where the FCNC contributions can be computed in terms of a very limited set of unknown new SUSY parameters. Remarkably enough, this minimal model succeeds to pass all the set of FCNC tests unscathed. To be sure, it is possible to severely constrain the SUSY parameter space, for instance using $b \rightarrow s \gamma$ in a way which is complementary to what is achieved by direct SUSY searches at colliders.

However, the MSSM is by no means equivalent to low-energy SUSY. A first sharp distinction concerns the mechanism of SUSY breaking and transmission to the observable sector which is chosen. As we mentioned above, in models with gauge-mediated SUSY breaking it may be possible to avoid the FCNC threat "ab initio" (notice that this is not an automatic feature of this class of models, but it depends on the specific choice of the sector which transmits the SUSY breaking information, the so-called messenger sector). The other more "canonical" class of SUSY theories that was mentioned above has gravitational messengers and a very large scale at which SUSY breaking occurs. In this talk we will focus only on this class of gravity-mediated SUSY breaking models. Even sticking to this more limited choice we have a variety of options with 
very different implications for the flavour problem.

\section{Rare $B$ decays in the MSSM and beyond}

Although the name seems to indicate a well-defined particle model, actually MSSM denotes at least two quite different classes of low-energy SUSY models. In its most restrictive meaning it denotes the minimal SUSY extension of the SM (i.e. with the smallest needed number of superfields) with R-parity, radiative breaking of electroweak symmetry, universality of the soft breaking terms and simplifying relations at the GUT scale among SUSY parameters. In this "minimal" version the MSSM exhibits only four free parameters in addition to those of the SM. Moreover, some authors impose specific relations between the two parameters $A$ and $B$ that appear in the trilinear and bilinear scalar terms of the soft breaking sector, further reducing the number of SUSY free parameters to three. Then, all SUSY masses are just function of these few independent parameters and, hence, many relations among them exist. Obviously this very minimal version of the MSSM can be very predictive. The most powerful constraint on this minimal model in the FCNC context comes from $b \rightarrow s \gamma$.

In SUSY there are five classes of one-loop diagrams which contribute to FCNC $B$ processes. They are distinguished according to the virtual particles running in the loop: $\mathrm{W}$ and up-quarks, charged Higgs and up-quarks, charginos and up-squarks, neutralinos and down-squarks, gluinos and down-squarks. It turns out that, at least in this "minimal" version of the MSSM, the charged Higgs and chargino exchanges yield the dominant SUSY contributions. As for $b \rightarrow s \gamma$ the situation can be summarized as follows. The CLEO measurement $\mathrm{B}$ yields $\operatorname{BR}\left(B \rightarrow X_{s} \gamma\right)=(2.32 \pm 0.67) \times 10^{-4}$. On the theoretical side we have just witnessed a major breakthrough with the computation of the nextto-leading logarithmic result for the BR. This has been achieved thanks to the calculation of the $O\left(\alpha_{s}\right)$ matrix elements 4 and of the next-to-feading order Wilson coefficients at $\mu \simeq m_{b}$ 专 The result quoted by Buras et al. 6 is $\operatorname{BR}(B \rightarrow$ $\left.X_{s} \gamma\right)=(3.48 \pm 0.31) \times 10^{-4}$ in the SM. A substantial improvement also on the experimental error is foreseen for the near future. Hence $b \rightarrow s \gamma$ is going to constitute the most relevant place in FCNC $B$ physics to constrain SUSY at least before the advent of $B$ factories. So far this process has helped in ruling out regions of the SUSY parameter space which are even larger than those excluded by LEP I and it is certainly going to be complementary to what LEP II is expected to do in probing the SUSY parameter space. After the detailed analysis in 1991 for small values of $\tan \beta$, there have been recent analyses $\mathrm{l}$ covering the entire range of $\tan \beta$ and including also other technical 
improvements (for instance radiative corrections in the Higgs potential). It has been shown $\mathrm{l}$ that the exclusion plots are very sensitive also to the relation one chooses between A and B. It should be kept in mind that the "traditional" relation $B=A-1$ holds true only in some simplified version of the MSSM. A full discussion is beyond the scope of this talk and so we refer the interested readers to the vast literature which exists on the subject.

The constraint on the SUSY parameter space of the "minimal" version of the MSSM greatly affects also the potential departures of this model from the $\mathrm{SM}$ expectation for $b \rightarrow s \ell^{+} \ell^{-}$. The present limits $10-10$ on the exclusive channels $\operatorname{BR}\left(B^{0} \rightarrow K^{* 0} e^{+} e^{-}\right)_{C L E O}<1.6 \times 10^{-5}$ and $\operatorname{BR}\left(B^{0} \rightarrow K^{* 0} \mu^{+} \mu^{-}\right)_{C D F}<$ $2.1 \times 10^{-5}$ are within an order of magnitude of the SM predictions. On the theoretical side, it has been estimated that the evaluation of $\Gamma\left(B \rightarrow X_{s} \ell^{+} \ell^{-}\right)$ in the SM is going to be affected by an error which cannot be reduced to less than $10-20 \%$ due to uncertainties in quark masses and interference effects from excited charmonium states 12 It turns out that, keeping into account the bound on $b \rightarrow s \gamma$, in the MSSM with universal soft breaking terms a $20 \%$ departure from the SM expected BR is kind of largest possible value one can obtain 13 Hence the chances to observe a meaningful deviation in this case are quite slim. However, it has been stressed that in view of the fact that three Wilson coefficients play a relevant role in the effective low-energy Hamiltonian involved in $b \rightarrow s \gamma$ and $b \rightarrow s \ell^{+} \ell^{-}$, a third observable in addition to $\mathrm{BR}(b \rightarrow s \gamma)$ and $\mathrm{BR}\left(b \rightarrow s \ell^{+} \ell^{-}\right)$is needed. This has been identified in some asymmetry of the emitted leptons (see refs. $13-4$ for two different choices of such asymmetry). This quantity, even in the "minimal" MSSM, may undergo a conspicuous deviation from its SM expectation and, hence, hopes of some manifestation of SUSY, even in this minimal realization, in $b \rightarrow s \ell^{+} \ell^{-}$are still present.

Finally, also for the $B_{d}-\bar{B}_{d}$ mixing, in the above-mentioned analysis of rare $B$ physics in the MSSM with universal soft breaking terms 0 it was emphasized that, at least in the low $\tan \beta$ regime, one cannot expect an enhancement larger than $20 \%-30 \%$ over the SM prediction (see also ref. 15 ). Moreover it was shown that $x_{s} / x_{d}$ is expected to be the same as in the SM.

It should be kept in mind that the above stringent results strictly depend not only on the minimality of the model in terms of the superfields that are introduced, but also on the "boundary" conditions that are chosen. All the lowenergy SUSY masses are computed in terms of the $M_{P l}$ four SUSY parameters through the RGE evolution. If one relaxes this tight constraint on the relation of the low-energy quantities and treats the masses of the SUSY particles as independent parameters, then much more freedom is gained. This holds true even if flavour universality is enforced. For instance, $\operatorname{BR}(b \rightarrow s \gamma)$ and $\Delta m_{B_{d}}$ 
may vary a lot from the SM expectation, in particular in regions of moderate SUSY masses 16

Moreover, flavour universality is by no means a prediction of low-energy SUSY. The absence of flavour universality of soft-breaking terms may result from radiative effects at the GUT scale or from effective supergravities derived from string theory. In the non-universal case, $\mathrm{BR}\left(b \rightarrow s \ell^{+} \ell^{-}\right)$is strongly affected by this larger freedom in the parameter space. There are points of this parameter space where the nonresonant $\operatorname{BR}\left(B \rightarrow X_{s} e^{+} e^{-}\right)$and $\operatorname{BR}(B \rightarrow$ $\left.X_{s} \mu^{+} \mu^{-}\right)$are enhanced by up to $90 \%$ and $110 \%$ while still respecting the constraint coming from $b \rightarrow s \gamma 13$

\section{Model-independent analysis of FCNC processes in SUSY}

Given a specific SUSY model it is in principle possible to make a full computation of all the FCNC phenomena in that context. However, given the variety of options for low-energy SUSY which was mentioned in the Introduction (even confining ourselves here to models with $\mathrm{R}$ matter parity), it is important to have a way to extract from the whole host of FCNC processes a set of upper limits on quantities which can be readily computed in any chosen SUSY frame.

The best model-independent parameterization of FCNC effects is the socalled mass insertion approximation 17 It concerns the most peculiar source of FCNC SUSY contributions that do not arise from the mere supersymmetrization of the FCNC in the SM. They originate from the FC couplings of gluinos and neutralinos to fermions and sfermions 18 One chooses a basis for the fermion and sfermion states where all the couplings of these particles to neutral gauginos are flavour diagonal, while the FC is exhibited by the non-diagonality of the sfermion propagators. Denoting by $\Delta$ the off-diagonal terms in the sfermion mass matrices (i.e. the mass terms relating sfermions of the same electric charge, but different flavour), the sfermion propagators can be expanded as a series in terms of $\delta=\Delta / \tilde{m}^{2}$ where $\tilde{m}$ is the average sfermion mass. As long as $\Delta$ is significantly smaller than $\tilde{m}^{2}$, we can just take the first term of this expansion and, then, the experimental information concerning ECNC and CP violating phenomena translates into upper bounds on these $\delta$ 's 1920

Obviously the above mass insertion method presents the major advantage that one does not need the full diagonalization of the sfermion mass matrices to perform a test of the SUSY model under consideration in the FCNC sector. It is enough to compute ratios of the off-diagonal over the diagonal entries of the sfermion mass matrices and compare the results with the general bounds on the $\delta$ 's that we provide here from all available experimental information.

There exist four different $\Delta$ mass insertions connecting flavours $i$ and $j$ 
along a sfermion propagator: $\left(\Delta_{i j}\right)_{L L},\left(\Delta_{i j}\right)_{R R},\left(\Delta_{i j}\right)_{L R}$ and $\left(\Delta_{i j}\right)_{R L}$. The indices $L$ and $R$ refer to the helicity of the fermion partners. The size of these $\Delta$ 's can be quite different. For instance, it is well known that in the MSSM case, only the $L L$ mass insertion can change flavour, while all the other three above mass insertions are flavour conserving, i.e. they have $i=j$. In this case to realize a $L R$ or $R L$ flavour change one needs a double mass insertion with the flavour changed solely in a $L L$ mass insertion and a subsequent flavourconserving $L R$ mass insertion. Even worse is the case of a FC $R R$ transition: in the MSSM this can be accomplished only through a laborious set of three mass insertions, two flavour-conserving $L R$ transitions and an $L L F C$ insertion. Instead of the dimensional quantities $\Delta$ it is more useful to provide bounds making use of dimensionless quantities, $\delta$, that are obtained dividing the mass insertions by an average sfermion mass.

The FCNC processes in $B$ physics which provide the best bounds on the $\delta_{23}$ and $\delta_{13} \mathrm{FC}$ insertions are $b \rightarrow s \gamma$ and $B_{d}-\bar{B}_{d}$, respectively.

The process $b \rightarrow s \gamma$ requires a helicity flip. In the presence of a $\left(\delta_{23}^{d}\right)_{L R}$ mass insertion we can realize this flip in the gluino running in the loop. On the contrary, the $\left(\delta_{23}^{d}\right)_{L L}$ insertion requires the helicity flip to occur in the external $b$-quark line. Hence we expect a stronger bound on the $\left(\delta_{23}^{d}\right)_{L R}$ quantity. Indeed, this is what happens: $\left(\delta_{23}^{d}\right)_{L L}$ is essentially not bounded, while $\left(\delta_{23}^{d}\right)_{L R}$ is limited to be $<(1.3 \div 3) \times 10^{-2}$ for an average squark mass of $500 \mathrm{GeV}$ and $0.3<m_{\tilde{g}}^{2} / m_{\tilde{q}}^{2}<4.0$ (these bounds scale as $m_{\tilde{q}}^{2}$ ). Given the upper bound on $\left(\delta_{23}^{d}\right)_{L R}$ from $b \rightarrow s \gamma$, it turns out that the quantity $x_{s}$ of $B_{s}-\bar{B}_{s}$ mixing receives contributions from this kind of mass insertions which are very tiny. The only chance to obtain large values of $x_{s}$ is if $\left(\delta_{23}^{d}\right)_{L L}$ is large, say of $O(1)$. In that case $x_{s}$ can easily jump up to values of $O\left(10^{2}\right)$ or even larger.

As for the mixing $B_{d}-\bar{B}_{d}$, we obtain

$$
\begin{aligned}
\sqrt{\left|\operatorname{Re}\left(\delta_{13}^{d}\right)_{L L}^{2}\right|} & <4.6 \cdot 10^{-2} ; \\
\sqrt{\left|\operatorname{Re}\left(\delta_{13}^{d}\right)_{L R}^{2}\right|} & <5.6 \cdot 10^{-2} ; \\
\sqrt{\left|\operatorname{Re}\left(\delta_{13}^{d}\right)_{L L}\left(\delta_{13}^{d}\right)_{R R}\right|} & <1.6 \cdot 10^{-2} ;
\end{aligned}
$$

for $x \equiv m_{\tilde{g}}^{2} / m_{\tilde{q}}^{2}=0.3$ with $m_{\tilde{q}}=500 \mathrm{GeV}$. The above bounds scale with $m_{\tilde{q}}(\mathrm{GeV}) / 500$ for different values of $m_{\tilde{q}}$ (at fixed $x$ ).

Then, imposing the bounds (11), we can obtain the largest possible value for $\operatorname{BR}(b \rightarrow d \gamma)$ through gluino exchange. As expected, the $\left(\delta_{13}^{d}\right)_{L L}$ insertion leads to very small values of this $\mathrm{BR}$ of $O\left(10^{-7}\right)$ or so, whilst the $\left(\delta_{13}^{d}\right)_{L R}$ 
insertion allows for $\mathrm{BR}(b \rightarrow d \gamma)$ ranging from few times $10^{-4}$ un to few times $10^{-3}$ for decreasing values of $x=m_{\tilde{g}}^{2} / m_{\tilde{q}}^{2}$. In the $\mathrm{SM}$ we expect $21 \mathrm{BR}(b \rightarrow d \gamma)$ to be typically $10-20$ times smaller than $\operatorname{BR}(b \rightarrow s \gamma)$, i.e. $\operatorname{BR}(b \rightarrow d \gamma)=$ $(1.7 \pm 0.85) \times 10^{-5}$. Hence a large enhancement in the SUSY case is conceivable if $\left(\delta_{13}^{d}\right)_{L R}$ is in the $10^{-2}$ range. Notice that in the MSSM we expect $\left(\delta_{13}^{d}\right)_{L R}<$ $m_{b}^{2} / m_{\tilde{q}}^{2} \times V_{t d}<10^{-6}$, hence with no hope at all of a sizeable contribution to $b \rightarrow d \gamma$.

\section{Is it possible to disentangle SUSY from SM in CP-violating $B$ decays?}

Keeping on with the approach that tackles FCNC effects in a generic SUSY extension of the SM, we would like to address the following two questions in this section: i) how large are the uncertainties of the SM predictions for CP asymmetries in B decays? and ii) in which processes and how can one possibly distinguish SUSY from SM coptributions (without making any commitment to the particular SUSY model)? 22

Concerning the former above question we will work in the framework of the analysis of ref. 23 We use the effective Hamiltonian $\left(\mathcal{H}_{e f f}\right)$ formalism, including LO QCD corrections; in the numerical analysis, we fuse the LO SM Wilson coefficients evaluated at $\mu=5 \mathrm{GeV}$, as given in ref. 24 In most of the cases, by choosing different scales (within a resonable range) or by using NLO Wilson coefficients, the results vary by about $20-30 \%$. This is true with the exception of some particular channels where uncertainties are larger. The matrix elements of the operators of $\mathcal{H}_{\text {eff }}$ are given in terms of the following Wick contractions between hadronic states: Disconnected Emission $(D E)$, Connected Emission $(C E)$, Disconnected Annihilation $(D A)$, Connected Annihilation $(C A)$, Disconnected Penguin $(D P)$ and Connected Penguin $(C P)$ (either for left-left $(L L)$ or for left-right $(L R)$ current-current operators). Following ref.25, where a detailed discussion can be found, instead of adopting a specific model for estimating the different diagrams, we let them vary within reasonable ranges to estimate the SM uncertainty (see ref. 23). First, only $D E=D E_{L L}=D E_{L R}$ are assumed to be different from zero (for simplicity, unless stated otherwise, the same numerical values are used for diagrams corresponding to the insertion of $L L$ or $L R$ operators, i.e. $D E=D E_{L L}=D E_{L R}$, $C E=C E_{L L}=C E_{L R}$, etc.). We then consider, in addition to $D E$, the $C E$ contribution by taking $C E=D E / 3$. Anpihilation diagrams are then included, and we use $D A=0$ and $C A=1 / 2 D E 25$ Inspired by kaon decays, we allow for some enhancement of the matrix elements of left-right (LR) operators and choose $D E_{L R}=2 D E_{L L}$ and $C E_{L R}=2 C E_{L L}$. Penguin contractions, $C P$ and 
$D P$, can be interpreted as long-distance penguin contributions to the matrix elements and play an important role: if we take $C P_{L L}=C E$ and $D P_{L L}=D E$, in some decays these terms dominate the amplitude. Finally, we allow for long distance effects which might differentiate penguin contractions with up and charm quarks in the loop, giving rise to incomplete GIM cancellations (we assume $\overline{D P}=D P(c)-D P(u)=D E / 3$ and $\overline{C P}=C P(c)-C P(u)=C E / 3)$. For any given decay channel, whenever two terms with different CP phases contribute in the SM, letting the various matrix elements vary within the above ranges, we estimate the ratio $r_{S M}$ of the two amplitudes, which is reported in the fifth column of table 1 .

New physics changes $\mathrm{SM}$ predictions on $\mathrm{CP}$ asymmetries in $B$ decays in two ways: by shifting the phase of the $B_{d}-\bar{B}_{d}$ mixing amplitude and by modifying both phases and absolute values of the decay ones. The generic SUSY extension of the SM considered here affects all these quantities.

In the SUSY case, by using for the Wilson coefficients in eq. (12) the results of ref. 20 and by parameterizing the matrix elements as we did for the SM case discussed above, we obtain the ratios of SUSY to SM amplitudes given in table 1. For each decay channel we give results for squark and gluino masses of $250 \mathrm{GeV}$ ( $r_{250}$ in the sixth column). We remark that the inclusion of the various terms in the amplitudes, $D E, D A$, etc., can modify the ratio $r$ of SUSY to SM contributions up to one order of magnitude.

In terms of the decay amplitude $A$, the $\mathrm{CP}$ asymmetry reads

$$
\mathcal{A}(t)=\frac{\left(1-|\lambda|^{2}\right) \cos \left(\Delta M_{d} t\right)-2 \operatorname{Im} \lambda \sin \left(\Delta M_{d} t\right)}{1+|\lambda|^{2}}
$$

with $\lambda=e^{-2 i \phi^{M}} \bar{A} / A$. In order to be able to discuss the results modelindependently, we have labelled as $\phi^{M}$ the generic mixing phase. The ideal case occurs when one decay amplitude only appears in (or dominates) a decay process: the $\mathrm{CP}$ violating asymmetry is then determined by the total phase $\phi^{T}=\phi^{M}+\phi^{D}$, where $\phi^{D}$ is the weak phase of the decay. This ideal situation is spoiled by the presence of several interfering amplitudes. If the ratios $r$ in table 1 are small, then the uncertainty on the sine of the CP phase is $<r$, while if $r$ is $\mathrm{O}(1) \phi^{T}$ receives, in general, large corrections.

The results of our analysis are summarized in table 1 In the third column, we give the values for the BR's of the various channelse 26 obtained using QCD sum rules form factors 27 to evaluate $D E$, and $|C E|=0.46 \times D E$, fitted using the measured two-body $B$ decays. The range of values given corresponds to the variation of the CKM amgles and to the inclusion of the charming penguin contributions (see ref. 6 for further details). In the fourth column, for each channel, we give the possible SM decay phases when one or two decay 
amplitudes contribute, and the range of variation of their ratio, $r_{S M}$, as explained above. A few comments are necessary at this point: a) for $B \rightarrow K_{S} \pi^{0}$ the penguin contributions (with a vanishing phase) dominate over the treelevel amplitude because the latter is Cabibbo suppressed; b) for the channel $b \rightarrow s \bar{s} d$ only penguin operators or penguin contractions of current-current operators contribute; c) the phase $\gamma$ is present in the penguin contractions of the $(\bar{b} u)(\bar{u} d)$ operator, denoted as $u$-P $\gamma$ in table 1 ; d) $\bar{b} d \rightarrow \bar{q} q$ indicates processes occurring via annihilation diagrams which can be measured from the last two channels of table 1 ; e) in the case $B \rightarrow K^{+} K^{-}$both current-current and penguin operators contribute; f) in $B \rightarrow D^{0} \bar{D}^{0}$ the contributions from the $(\bar{b} u)(\bar{u} d)$ and the $(\bar{b} c)(\bar{c} d)$ current-current operators (proportional to the phase $\gamma)$ tend to cancel out.

SUSY contributes to the decay amplitudes with phases induced by $\delta_{13}$ and $\delta_{23}$ which we denote as $\phi_{13}$ and $\phi_{23}$. The ratio $A_{S U S Y} / A_{S M}$ for SUSY masses of $250 \mathrm{GeV}$ is reported in the $r_{250}$ column of table 1 .

We now draw some conclusions from the results of table 1 . In the SM, the first six decays measure directly the mixing phase $\beta$, up to corrections which, in most of the cases, are expected to be small. These corrections, due to the presence of two amplitudes contributing with different phases, produce uncertainties of $\sim 10 \%$ in $B \rightarrow K_{S} \pi^{0}$, and of $\sim 30 \%$ in $B \rightarrow D^{+} D^{-}$and $B \rightarrow J / \psi \pi^{0}$. In spite of the uncertainties, however, there are cases where the SUSY contribution gives rise to significant changes. For example, for SUSY masses of $\mathrm{O}(250) \mathrm{GeV}$, SUSY corrections can shift the measured value of the sine of the phase in $B \rightarrow \phi K_{S}$ and in $B \rightarrow K_{S} \pi^{0}$ decays by an amount of about $70 \%$. For these decays SUSY effects are sizeable even for masses of 500 $\mathrm{GeV}$. In $B \rightarrow J / \psi K_{S}$ and $B \rightarrow \phi \pi^{0}$ decays, SUSY effects are only about $10 \%$ but SM uncertainties are negligible. In $B \rightarrow K^{0} \bar{K}^{0}$ the larger effect, $\sim 20 \%$, is partially covered by the indetermination of about $10 \%$ already existing in the SM. Moreover the rate for this channel is expected to be rather small. In $B \rightarrow$ $D^{+} D^{-}$and $B \rightarrow K^{+} K^{-}$, SUSY effects are completely obscured by the errors in the estimates of the SM amplitudes. In $B^{0} \rightarrow D_{C P}^{0} \pi^{0}$ the asymmetry is sensitive to the mixing angle $\phi_{M}$ only because the decay amplitude is unaffected by SUSY. This result can be used in connection with $B^{0} \rightarrow K_{s} \pi^{0}$, since a difference in the measure of the phase is a manifestation of SUSY effects.

Turning to $B \rightarrow \pi \pi$ decays, both the uncertainties in the SM and the SUSY contributions are very large. Here we witness the presence of three independent amplitudes with different phases and of comparable size. The observation of SUSY effects in the $\pi^{0} \pi^{0}$ case is hopeless. The possibility of separating SM and SUSY contributions by using the isospin analysis remains an open possibility which deserves further investigation. For a thorough discussion of the SM 
Table 1: Branching ratios and CP phases for B decays. $\phi_{S M}^{D}$ denotes the decay phase in the SM; T and P denote Tree and Penguin, respectively; for each channel, when two amplitudes with different weak phases are present, one is given in the first row, the other in the last one and the ratio of the two in the $r_{S M}$ column. $\phi_{S U S Y}^{D}$ denotes the phase of the SUSY amplitude, and the ratio of the SUSY to SM contributions is given in the $r_{250}$ column.

\begin{tabular}{|c|c|c|c|c|c|c|}
\hline Incl. & Excl. & $\mathrm{BR} \times 10^{5}$ & $\phi_{\mathrm{SM}}^{D}$ & $r_{\mathrm{SM}}$ & $\phi_{\mathrm{SUSY}}^{D}$ & $r_{250}$ \\
\hline$b \rightarrow c \bar{c} s$ & $B \rightarrow J / \psi K_{S}$ & 40 & 0 & - & $\phi_{23}$ & $0.03-0.1$ \\
\hline$b \rightarrow s \bar{s} s$ & $B \rightarrow \phi K_{S}$ & $0.6-2$ & 0 & - & $\phi_{23}$ & $0.4-0.7$ \\
\hline \multirow[t]{2}{*}{$b \rightarrow u \bar{u} s$} & & & $\mathrm{P} 0$ & & & \\
\hline & $B \rightarrow \pi^{0} K_{S}$ & $0.02-0.4$ & & $0.01-0.08$ & $\phi_{23}$ & $0.4-0.7$ \\
\hline$b \rightarrow d \bar{d} s$ & & & $\mathrm{~T} \gamma$ & & & \\
\hline \multirow[t]{2}{*}{$b \rightarrow c \bar{u} d$} & & & 0 & & & \\
\hline & $B \rightarrow D_{C P}^{0} \pi^{0}$ & 16 & & 0.02 & - & - \\
\hline \multicolumn{2}{|l|}{$b \rightarrow u \bar{c} d$} & & $\gamma$ & & & \\
\hline \multirow{3}{*}{$b \rightarrow c \bar{c} d$} & $B \rightarrow D^{+} D^{-}$ & $30-50$ & T 0 & $0.03-0.3$ & & $0.007-0.02$ \\
\hline & & & & \multicolumn{3}{|c|}{$\phi_{13}$} \\
\hline & $B \rightarrow J / \psi \pi^{0}$ & 2 & $\mathrm{P} \beta$ & $0.04-0.3$ & & $0.007-0.03$ \\
\hline \multirow{3}{*}{$b \rightarrow s \bar{s} d$} & $B \rightarrow \phi \pi^{0}$ & $1-4 \times 10^{-4}$ & $\mathrm{P} \beta$ & - & \multirow{3}{*}{$\phi_{13}$} & $0.06-0.1$ \\
\hline & & & & & & \\
\hline & $B \rightarrow K^{0} \bar{K}^{0}$ & $0.007-0.3$ & $u-\mathrm{P} \gamma$ & $0-0.07$ & & $0.08-0.2$ \\
\hline \multirow{3}{*}{$\begin{array}{r}b \rightarrow u \bar{u} d \\
b \rightarrow d \bar{d} d\end{array}$} & $B \rightarrow \pi^{+} \pi^{-}$ & $0.2-2$ & $\mathrm{~T} \gamma$ & $0.09-0.9$ & \multirow{2}{*}{$\begin{array}{l}\phi_{13} \\
\phi_{13}\end{array}$} & $0.02-0.8$ \\
\hline & $B \rightarrow \pi^{0} \pi^{0}$ & $0.003-0.09$ & $\mathrm{P} \beta$ & $0.6-6$ & & $0.06-0.4$ \\
\hline & $B \rightarrow K^{+} K^{-}$ & $<0.5$ & $\mathrm{~T} \gamma$ & $0.2-0.4$ & \multirow{3}{*}{$\phi_{13}$} & $0.04-0.1$ \\
\hline \multirow[t]{2}{*}{$b \bar{d} \rightarrow q \bar{q}$} & & & & & & \\
\hline & $B \rightarrow D^{0} \bar{D}^{0}$ & $<20$ & $\mathrm{P} \beta$ & only $\beta$ & & $0.01-0.03$ \\
\hline
\end{tabular}

uncertainties in $B \rightarrow \pi \pi$ see ref. 25

In conclusion, our analysis shows that measurements of CP asymmetries in several channels may allow the extraction of the $\mathrm{CP}$ mixing phase and to disentangle SM and SUSY contributions to the CP decay phase. The goldenplated decays in this respect are $B \rightarrow \phi K_{S}$ and $B \rightarrow K_{S} \pi^{0}$ channels. The size of the SUSY effects is clearly controlled by the the non-diagonal SUSY mass insertions $\delta_{i j}$, which for illustration we have assumed to have the maximal value compatible with the present experimental limits on $B_{d}^{0}-\bar{B}_{d}^{0}$ mixing. 


\section{Acknowledgements}

We thank M. Ciuchini, E. Franco, F. Gabbiani, E. Gabrielli and G. Martinelli who collaborated with us in the model-independent analyses that we presented here. We wish to thank the organizers of $B$ Physics and CP Violation Conference for giving us the opportunity of discussing $B$ physics in a stimulating and pleasant atmosphere. The work of A. M. was partially supported by the EU contract ERBFMRX CT96 0090. L. S. acknowledges the support of Fondazione Angelo della Riccia, Firenze.

\section{References}

[1] J. Ellis and D.V. Nanopoulos, Phys. Lett. B 110, 44 (1982);

R. Barbieri and R. Gatto, Phys. Lett. B 110, 211 (1982).

[2] For a phenomenologically oriented review, see:

P. Fayet and S. Ferrara, Phys. Rep. 32 C, 249 (1977);

H.P. Nilles, Phys. Rep. 110 C, 1 (1984).

H.E. Haber and G.L Kane, Phys. Rep. 117 C, 1 (1987);

For spontaneously broken $\mathrm{N}=1$ supergravity, see:

E. Cremmer, S. Ferrara, L. Girardello and A. Van Proeyen, Nucl. Phys. B 212, 413 (1983) and references therein.

P. Nath, R. Arnowitt and A.H. Chamseddine, Applied N=1 Supergravity ( World Scientific, Singapore, 1984);

A.G. Lahanas and D.V. Nanopoulos, Phys. Rep. 145 C, 1 (1987).

[3] M.S. Alam et al. (CLEO collab.), Phys. Rev. Lett. 74, 2885 (1995).

[4] C. Greub, T. Hurth and D. Wyler, Phys. Lett. B 380, 385 (1996); Phys. Rev. D 54, 3350 (1996).

[5] K.G. Chetyrkin, M. Misiak and M. Münz, Phys. Lett. B 400, 206 (1997).

[6] A.J. Buras, A. Kwiatkowski and N. Pott, Preprint TUM-HEP-287/97, hep-ph/9707482.

[7] S. Bertolini, F. Borzumati, A. Masiero and G. Ridolfi, Nucl. Phys. B 353, 591 (1991).

[8] R. Barbieri and G.F. Giudice, Phys. Lett. B 309, 86 (1993);

N. Oshimo, Nucl. Phys. B 404, 20 (1993);

R. Garisto and J.N. Ng, Phys. Lett. B 315, 372 (1993);

M.A. Diaz, Phys. Lett. B 304, 278 (1993);

Y. Okada, Phys. Lett. B 315, 119 (1993);

F. Borzumati, Z. Phys. C 63, 291 (1994);

P. Nath and R. Arnowitt, Phys. Lett. B 336, 395 (1994);

S. Bertolini and F. Vissani, Z. Phys. C 67, 513 (1995);

J. Lopez et al., Phys. Rev. D 51, 147 (1995). 
[9] S. Bertolini and F. Vissani, in ref.

[10] R. Balest et al. (CLEO collab.), CLEO-CONF 94-4 (1994).

[11] C. Anway-Wiese et al. (CDF collab), Fermilab-Conf-95/201-E (1995).

[12] Z. Ligeti and M.B. Wise, Phys. Rev. D 53, 4937 (1996).

[13] P. Cho, M. Misiak and D. Wyler, Phys. Rev. D 54, 3329 (1996).

[14] A. Ali, G.F. Giudice and T. Mannel, Z. Phys. C 67, 417 (1995).

[15] T. Kurimoto, Phys. Rev. D 39, 3447 (1989).

[16] G.C. Branco, G.C. Cho, Y. Kizukuri and N. Oshimo, Phys. Lett. B 337, 316 (1994); Nucl. Phys. B 44, 483 (1995);

A. Brignole, F. Feruglio and F. Zwirner, Z. Phys. C 71, 679 (1996).

[17] L.J. Hall, V.A. Kostelecky and S. Raby, Nucl. Phys. B 267, 415 (1986).

[18] M.J. Duncan, Nucl. Phys. B 221, 285 (1983);

J.F. Donoghue, H.P. Nilles and D. Wyler, Phys. Lett. B 128, 55 (1983);

A. Bouquet, J. Kaplan and C.A. Savoy, Phys. Lett. B 148, 69 (1984).

[19] F. Gabbiani and A. Masiero, Nucl. Phys. B 322, 235 (1989);

J.S. Hagelin, S. Kelley and T. Tanaka, Nucl. Phys. B 415, 293 (1994);

E. Gabrielli, A. Masiero and L. Silvestrini, Phys. Lett. B 374, 80 (1996).

[20] F. Gabbiani, E. Gabrielli, A. Masiero and L. Silvestrini, Nucl. Phys. B 477, 321 (1996).

[21] A. Ali, Nucl. Instrum. Meth. A384, 8 (1996).

[22] For some recent attempts to tackle this latter crucial question, see:

N. Deshpande, B. Dutta and S. Oh, Phys. Rev. Lett. 77, 4499 (1996);

J. Silva and L. Wolfenstein, Phys. Rev. D 53, 5331 (1997);

A. Cohen, D. Kaplan, F. Leipentre and A. Nelson, Phys. Rev. Lett. 78, 2300 (1997);

Y. Grossman and M. Worah, Phys. Lett. B 395, 241 (1997);

M. Ciuchini, E. Franco, G. Martinelli, A. Masiero and L. Silvestrini, Phys. Rev. Lett. 79, 978 (1997);

Y. Grossman, Y. Nir and M. Worah, hep-ph/9704287;

R. Barbieri and A. Strumia, hep-ph/9704402.

[23] M. Ciuchini, E. Franco, G. Martinelli, A. Masiero and L. Silvestrini, in ref. 22

[24] M. Ciuchini, E. Franco, G. Martinelli, L. Reina and L. Silvestrini, Z. Phys. C 68, 239 (1995).

[25] M. Ciuchini, E. Franco, G. Martinelli and L. Silvestrini, Preprint CERNTH/97-30, March 1997, hep-ph/9703353.

[26] M. Ciuchini, R. Contino, E. Franco, G. Martinelli and L. Silvestrini, Preprint CERN-TH/97-188, July 1997, hep-ph/9708222.

[27] V.M. Belayev et al., Phys. Rev. D 51, 6177 (1995);

P. Ball, Phys. Rev. D 48, 3190 (1993); 
P. Ball and V.M. Braun, Phys. Rev. D 54, 2182 (1996). 